\title{
A Proposed Model for MC-CDMA Based In-Place Wavelet Transform
}

\author{
Salih M. Salih \\ Electrical Department \\ College of Engineering \\ University of Al-Anbar/ Iraq \\ 009647901255958 \\ dr_salih_moh@yahoo.com
}

\author{
N. Uzunoglu \\ Electrical and Computer \\ Engineering Department/ \\ NTUA/ Greece \\ 00306977008922 \\ nnap@otenet.gr
}

\author{
L.Awda. Kadhim \\ Electrical and Electronic \\ Department/ University of \\ Technology/ Iraq \\ 009647901960913 \\ laithawda@yahoo.com
}

\author{
L.A.El_Anzy \\ Electrical Engineering \\ Department/ Al-Mousaib \\ Technical College/ Iraq \\ 009647801080458
}

\begin{abstract}
In this paper, a proposed model for Multicarrier-Code Division Multiple Access (MC-CDMA) lying in In-Place Wavelet Transform (IP-WT) algorithm was introduced and analyzed under the Additive White Gaussian Noise (AWGN) channel, flat fading channel and frequency selective fading channel. The performance of the proposed system was compared with the traditional model of MC-CDMA based Fast Fourier Transform (FFT). The proposed model does not need an additional array at each sweep such as the ordered fast Haar wavelet transform, this property will reduce the processing time and the memory size. The results show that the proposed model has an active performance under different channel characteristics.
\end{abstract}

\section{General Terms}

Algorithms, Performance, Design.

\section{Keywords}

MC-CDMA, Wavelet Transform, In-Place, Flat Fading, Frequency Selective.

\section{INTRODUCTION}

Recent studies by researchers have combined the principle of CDMA with Orthogonal Frequency Division Multiplexing (OFDM) which allows one to use the available spectrum in an efficient way and retain the many advantages of a CDMA system if the number of spacing between subcarriers is chosen appropriately, it is unlikely that all the subcarriers will be in deep fade and thus provides frequency diversity [1]. This combination of OFDM-CDMA is a useful technique for $4 \mathrm{G}$ systems, which has the property of variable data rates as well as provides reliable communication systems. In OFDM based wavelet transform, the IFFT and FFT blocks are simply replaced by an Inverse Discrete Wavelet Transform (IDWT) and Discrete Wavelet Transform (DWT), respectively [2]. Due to the higher spectral containment between subchannels, wavelet-based OFDM can be better in combating narrowband interference and is inherently more robust with respect to Inter-Carrier Interference (ICI) than traditional Fourier filters. Wavelets OFDM is implemented via overlapped waveforms to preserve data rate. The classic notion of a cyclic prefix does not make sense in this context. Without the cyclic prefix, the data rate in wavelet systems can surpass those of Fourier implementations; one of its key motivating factors. So, the main and important difference between FFT and discrete wavelet transform based OFDM is that the wavelet based OFDM will not add a cyclic prefix to OFDM symbol [2, 3]. This property makes the wavelet to compete with FFT for the future $4 \mathrm{G}$ system.

Permission to make digital or hard copies of all or part of this work for personal or classroom use is granted without fee provided that copies are not made or distributed for profit or commercial advantage and that copies bear this notice and the full citation on the first page. To copy otherwise, to republish, to post on servers or to redistribute to lists, requires prior specific permission and/or a fee. MOBIMEDIA 2007, August 27-29, Nafpaktos, Greece

(C)

\section{IN-PLACE WAVELET TRANSFORM}

The basic Haar transform expresses the approximating function with wavelets by replacing an adjacent pair of steps via one wider step and one wavelet. The wider step measures the average of the initial pair of steps, while the wavelet, formed by two alternating steps, measures the difference of the initial pair of steps. The ordered fast Haar wavelet transform requires additional arrays at each sweep, and it assumes that the whole sample is known at the start of the algorithm. In contrast, some applications require realtime processing as the signal proceeds, which precludes any knowledge of the whole sample, and some applications involve arrays so large that it does not allow sufficient space for additional arrays at each sweep. The two problems just described, lack of time or space, have a common solution in the In-Place Fast Haar wavelet transform [4], which differs from ordered fast Haar algorithm only in its indexing scheme.

\section{- In-Place Basic Sweep}

For each pair $a_{2 k}^{(n-[l-1])}, a_{2 k+1}^{(n-[l-1])}$, instead of placing its results in two additional arrays, the $l^{\text {th }}$ sweep of the in-place transform merely replaces the pair $a_{2 k}^{(n-[1-1])}, a_{2 k+1}^{(n-[l-1])}$ by the new entries $a_{k}^{(n-1)}, c_{k}^{(n-1)}$ :

Initialization: Consider the pair $a_{2 k}^{(n-[l-1])}, a_{2 k+1}^{(n-[l-1])}$.

Calculation: Perform the basic transform

$$
\begin{aligned}
& a_{k}^{(n-l)}=\frac{a_{2 k}^{(n-[l-1])}+a_{2 k+1}^{(n-[l-1])}}{2} \\
& c_{k}^{(n-l)}=\frac{a_{2 k}^{(n-[l-1])}-a_{2 k+1}^{(n-[l-1])}}{2}
\end{aligned}
$$

Replacement: Replace the initial pair $a_{2 k}^{(n-[l-1])}, a_{2 k+1}^{(n-[l-1])}$ by the transform $a_{k}^{(n-l)}, c_{k}^{(n-l)}$.

\subsection{In-Place Wavelet Transform Analysis}

The In-Place basic sweep explained in the preceding section extends to a complete algorithm through mere record-keeping. The first few sweeps proceed as follows

- Initialization

$$
\vec{S}^{(n-1)}=\vec{S}=\left(S_{0}, S_{1}, S_{2}, \ldots \ldots \ldots, S_{2 k}, S_{2 k+1}, \ldots \ldots \ldots S_{2^{n}-2}, S_{2^{n}-1}\right)
$$

- First Sweep

$$
\begin{aligned}
& \vec{S}^{(n-1)}=\left(\frac{S_{0}+S_{1}}{2}, \frac{S_{0}-S_{1}}{2}, \frac{S_{2}+S_{3}}{2}, \frac{S_{2}-S_{3}}{2}, \ldots \ldots \ldots \ldots ., \frac{S_{2 k}+S_{2 k+1}}{2}, \frac{S_{2 k}-S_{2 k+1}}{2}, \ldots \ldots\right. \\
& \left.\ldots \ldots . ., \frac{S_{2^{n}-2}}{2}+S_{2^{n}-1}, \frac{S_{2^{n}-2}-S_{2^{n}-1}}{2}\right) \\
& =\left(\mathbf{a}_{0}^{(n-1)}, c_{0}^{(n-1)}, \mathbf{a}_{1}^{(n-1)}, c_{1}^{(n-1)}, \mathbf{a}_{2}^{(n-1)}, c_{2}^{(n-1)}, \mathbf{a}_{3}^{(n-1)}, c_{3}^{(n-1)}, \ldots . .\right. \\
& \ldots \ldots ., \mathbf{a}_{k}^{(n-1)}, c_{k}^{(n-1)}, \ldots \ldots \ldots \ldots \ldots . . . \mathbf{a}_{2^{n-1}-1}^{(n-1)}, c_{2^{n-1}-1}^{(n-1)}
\end{aligned}
$$




\section{- Second Sweep}

In the new array $\vec{S}^{(n-1)}$, keep but skip over the wavelet coefficients $c_{k}^{(n-l)}$, and perform the basic sweep on the array $\mathbf{a}_{k}^{(n-1)}$

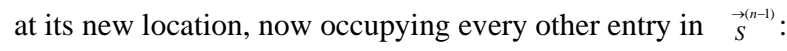

$$
\begin{aligned}
& \stackrel{-}{S}^{(n-2)}=\left(\frac{\mathbf{a}_{0}^{(n-1)}+\mathbf{a}_{1}^{(n-1)}}{2}, c_{0}^{(n-1)}, \frac{\mathbf{a}_{0}^{(n-1)}-\mathbf{a}_{1}^{(n-1)}}{2}, c_{1}^{(n-1)}, \frac{\mathbf{a}_{-}^{(n-1)}+\mathbf{a}_{3}^{(n-1)}}{2}, c_{2}^{(n-1)}, \frac{\mathbf{a}_{2}^{(n-1)}-\mathbf{a}_{3}^{(n-1)}}{2}, c_{3}^{(n-1)},\right. \\
& \left.\ldots . . \cdot \frac{\mathbf{a}_{2^{n-1}-2}^{(n-1)}+\mathbf{a}_{2^{n-1}-1}^{(n-1)}}{2}, c_{2^{-1-1}-2}^{(n-1)}, \frac{\mathbf{a}_{2^{n-1}-2}^{(n-1)}-\mathbf{a}_{2^{n-1}-1}^{(n-1)}}{2}, c_{2^{2^{-1}-1}}^{(n-1)}\right) \\
& =\left(\mathbf{a}_{0}^{(n-2)}, c_{0}^{(n-1)}, c_{0}^{(n-2)}, c_{1}^{(n-1)}, \mathbf{a}_{1}^{(n-2)}, c_{2}^{(n-1)}, c_{1}^{(n-2)}, c_{3}^{(n-1)}\right. \\
& \left., \mathbf{a}_{2}^{(n-2)}, C_{4}^{(n-1)}, C_{2}^{(n-2)}, C_{5}^{(n-1)}, \ldots \ldots \ldots \ldots \ldots C_{2^{n-2}-1}^{(n-2)}, C_{2^{n-1}-1}^{(n-1)}\right)
\end{aligned}
$$

In general, the In-Place $l^{\text {th }}$ sweep begins with an array

$$
\stackrel{\rightarrow(n-[l-1])}{S}=\left(\mathbf{a}_{0}^{(n-[l-1]}, c_{0}^{(n-1)}, c_{0}^{(n-2)}, c_{1}^{(n-1)}, c_{0}^{(n-3)}, c_{2}^{(n-1)}, c_{1}^{(n-2)}, c_{3}^{(n-1)}, \ldots . ., c_{2^{n-2}-1}^{(n-2)}, c_{2^{n-1}-1}^{(n-1)}\right.
$$

This contains the array

$$
\mathbf{a}^{(n-[l-1])}=\left(\mathbf{a}_{0}^{(n-[l-1])}, \mathbf{a}_{1}^{(n-[l-1])}, \ldots \ldots \ldots ., \mathbf{a}_{2^{n-(l-1)}-1}^{(n-[l-1])}\right)
$$

At the locations $\mathbf{a}_{k}^{(n-[l-1])}=S_{2^{l-1} k}^{(n-[l-1])}$, in other words, at multiples of $2^{l-1}$ apart in $\quad S_{S}^{(n-l l-1)}$, and which the $l^{\text {th }}$ sweep replaces by

$$
\begin{aligned}
& a_{j}^{(n-l)}=\frac{a_{2 j}^{(n-[l-1])}+a_{2 j+1}^{(n-[l-1])}}{2}=\frac{S_{l^{-1-1} 2 j}^{(n-[l-1])}+a_{2^{l-1}(2 j+1)}^{(n-l l-1])}}{2} \\
& c_{j}^{(n-l)}=\frac{a_{2 j}^{(n-[l-1])}+a_{2 j+1}^{(n-[l-1])}}{2}=\frac{S_{2^{-l}-2 j}^{(n-[l-1])}+a_{2^{l^{-1}(2 j+1)}}^{(n-[l-1])}}{2} \\
& S_{2^{l-1} 2 j}^{(n-l)}=a_{j}^{(n-l)}, \quad S_{2^{l-1}(2 j+1)}^{(n-l)}=c_{j}^{(n-l)}
\end{aligned}
$$

So that the new array $a^{\rightarrow(n-l)}$ occupies entries at multiples of $2^{l}$ apart in $\stackrel{\rightarrow(n-l)}{S}$, becomes $a_{j}^{(n-l)}=S_{2^{-1} 2 j}^{(n-l)}=S_{2^{-} j}^{(n-l)}$.

\subsection{The In-Place Fast Inverse Wavelet Transform Analysis (IP-IWT)}

As described in the preceding section, the fast Haar wavelet transform neither alters nor diminishes the information contained in the initial array $\vec{S}=\left(S_{0}, S_{1}, \ldots \ldots \ldots . ., S_{2^{n}-1}\right)$, because each basic transform

$$
a_{k}^{(l)}=\frac{a_{2 k}^{(n-1)}+a_{2 k+1}^{(n-1)}}{2}, \quad c_{k}^{(l)}=\frac{a_{2 k}^{(n-1)}-a_{2 k+1}^{(n-1)}}{2}
$$

admits an inverse transform:

$$
a_{2 k}^{(l-1)}=a_{k}^{l}+c_{k}^{l}, \quad a_{2 k+1}^{(l-1)}=a_{k}^{l}-c_{k}^{l} .
$$

Repeat the applications of the basic inverse transform just given, beginning with the wavelet coefficients

$$
\vec{S}^{(0)}=\left(a_{0}^{(n)}, c_{0}^{(1)}, \ldots \ldots \ldots . . ., c_{2^{n}-1}^{(1)}\right),
$$$$
\text { Reconstruct the initial array } \quad \vec{S}^{(n)}=\vec{S}=\left(S_{0}, S_{1} \ldots \ldots \ldots, S_{2^{n}-1}\right)
$$

\section{SIMULATION MODEL}

In this section, the proposed MC-CDMA transceiver based on InPlace Haar wavelet transform will be described, and its performance will be discussed. Fig. (1), shows the traditional MCCDMA based FFT and the proposed model is shown in fig. (2). It can be seen that the IFFT and FFT blocks in fig. (1) are replaced by the In-Place Inverse Wavelet Transform (IP-IWT) and the InPlace Wavelet Transform (IP-WT) blocks as in fig. (2).

Let us consider an input data binary sequence generated at the transmitter side of logic ' 1 ' with a Walsh-Hadamard spreading code number 20 for this simulation. The spreading signal is processed by the IP-IWT $(\mathrm{N}=32)$. The transmitted signal is subjected to random noise variation, Fig. 3 describes these steps. Also the training signal is generated with a 32 bit and processed by IP-IWT with a 32 bit zero padding and without spreading. The subplot $(3,3,1)$ represents the generated random signal which was taken as logic ' 1 ' in this plot, then spread the signal by Walsh code of 32 bit as in subplot $(3,3,2)$, the zeros are padded from bit $17-48$ in this simulation as in subplot $(3,3,3)$. Now, it is assumed that the signal transmitted through the channel to the receiver with an amplitude values shown in subplot $(3,3,4)$, this signal was fluctuated randomly due to the channel effect as shown in subplot $(3,3,5)$. After processing the signal by IP-WT, the fluctuation and the random distribution of signal was reduced (subplot $(3,3,6)$ ). Finally, the channel estimation and compensation are done on the data vector after removing the zeros that was added at the transmitter side.

The training sequence will be used to estimate the channel frequency response as follows $[5,6,7]$ :

$$
H(k)=\frac{\text { Received Training Sample }(k)}{\text { Transmitted Training Sample }(k)}
$$

The channel frequency response will be used to compensate the channel effects on the data, and the estimated data can be found using the following equation:

$$
\text { Estmate. data }=H^{-1} \text { estimate }(k) * \text { Re ceived.data }(k)
$$

Finally the signal is Exclusive Ored (XOR) with a WalshHadamard of the same user specific code at the transmitter side, and the detection threshold decision is used to decide the value of signal.

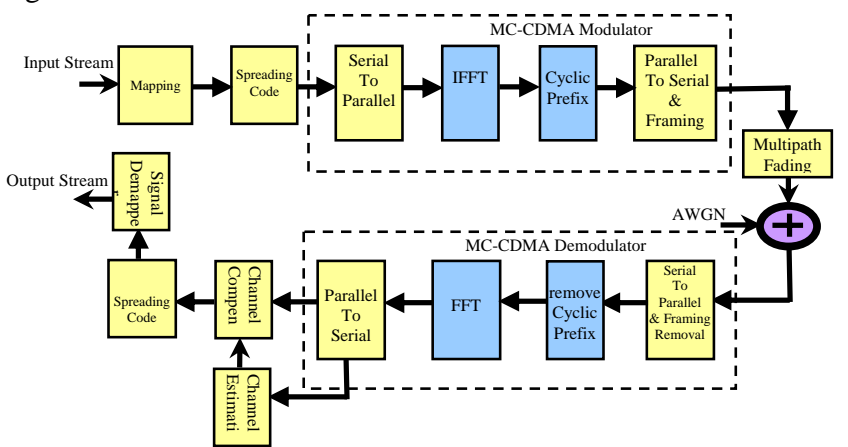

Figure 1. Traditional MC-CDMA model based FFT

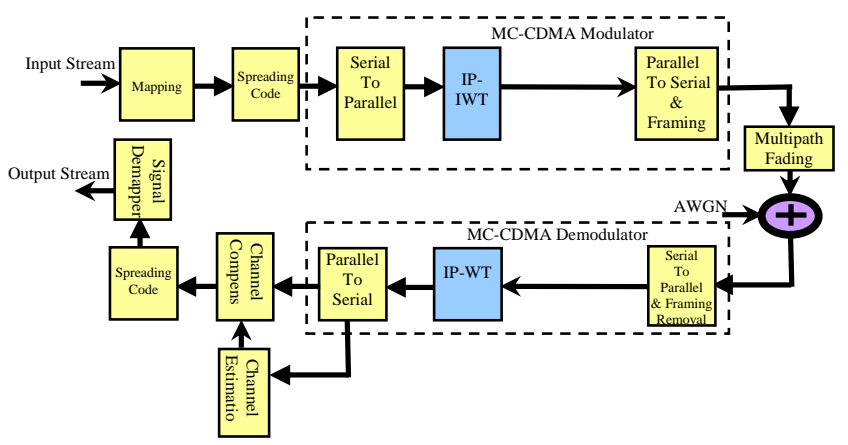

Figure 2. A proposed MC-CDMA model based In-Place wavelet transform 


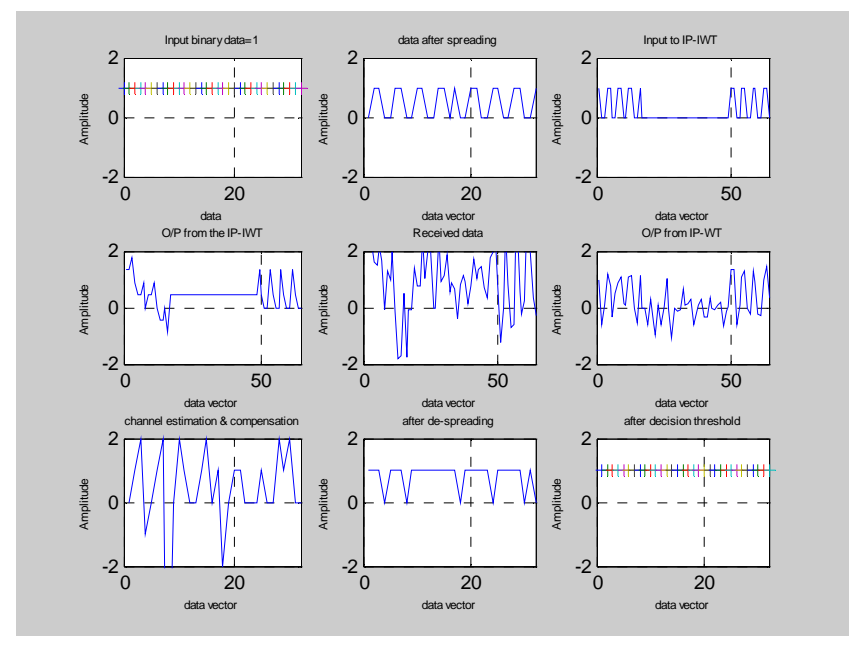

Figure 3. Time envelope of the proposed model

Hint: according to the nature distribution of the IP-WT, which may cause an increasing in the amplitude of the transmitted signal to be more than the level of the random noise generated by the Matlab tool as in subplot $(3,3,4)$, so, the transmitted mean value is set at 0.5 or less. This signal is scaled to be 0.5 by dividing it on its (absolute mean value*2). The output mean value of the transmitted signal or training is normalized to 0.5 as given by Eqs. (14) and (15).

AbsoluteMeanValue $_{\text {Dator Training }}(k)=\left(\operatorname{Real}_{k}{ }^{2}+\operatorname{Imag}_{k}{ }^{2}\right)^{1 / 2}, \quad k=1,2,3, \ldots \ldots . ., N$
Transmittel Symbol
Data and Training

At the receiver the signal and training are multiplied by $(2 *$ mean value) that are divided by it at the transmitter side.

\section{SIMULATION RESULTS}

In this section, the combination of conventional MC-CDMA with the proposed MC-CDMA based on IP-WT will be studied, in this research the Walsh-Hadamard (code 20) has been used with 32 bits of zeros are added. A simulation of the two systems has been made using MATLAB 7. The BER performance of the two systems will also be studied in different channel models which are AWGN, AWGN+flat fading and AWGN+frequency selective fading channel, with a bit rate of 5 Mbps and 64 subcarriers are used in this simulation.

\subsection{Performance of the Proposed System in AWGN Channel}

The channel here is modeled as an Additive White Gaussian Noise for wide range of SNR from $0 \mathrm{~dB}$ to $40 \mathrm{~dB}$, from Fig.(4), it is found that the proposed system of MC-CDMA based on IP-WT worked with $\mathrm{SNR}=9.5 \mathrm{~dB}$ at $\mathrm{BER}=10^{-4}$, while in the traditional MC-CDMA the SNR value at $B E R=10^{-4}$ is $23 \mathrm{~dB}$, which means a gain of $13.5 \mathrm{~dB}$ is obtained by the proposed model.

\subsection{Performance of the Proposed System in Flat Fading Channel}

The simulation results for both systems in flat fading channel are shown in fig.(5). Three values of the Doppler frequencies (fd) are considered in this simulation, these are $\mathrm{fd}=5 \mathrm{~Hz}, 500 \mathrm{~Hz}$ and 1100 $\mathrm{Hz}$ respectively. From this figure it can be seen that the proposed
MC-CDMA it still performs better than the traditional MCCDMA in all values of the Doppler frequencies, where it approaches the $\mathrm{BER}=10^{-4}$ at $\mathrm{SNR}=31 \mathrm{~dB}$ and $36 \mathrm{~dB}$ when the Doppler frequencies are $5 \mathrm{~Hz}$ and $500 \mathrm{~Hz}$. The BER increases as the Doppler frequency increases in both models. The traditional MC-CDMA is less sensitive to the variation of the Doppler frequency than the proposed one. For a $B E R=10^{-2}$, the $S N R=15$, 16 , and $16.5 \mathrm{~dB}$ for the proposed model with 33,34 , and $34.5 \mathrm{~dB}$ for the traditional system, which means the gain is more than 18 $\mathrm{dB}$ was obtained from the proposed model at Doppler frequencies are 5, 500, and $1100 \mathrm{~Hz}$ respectively.

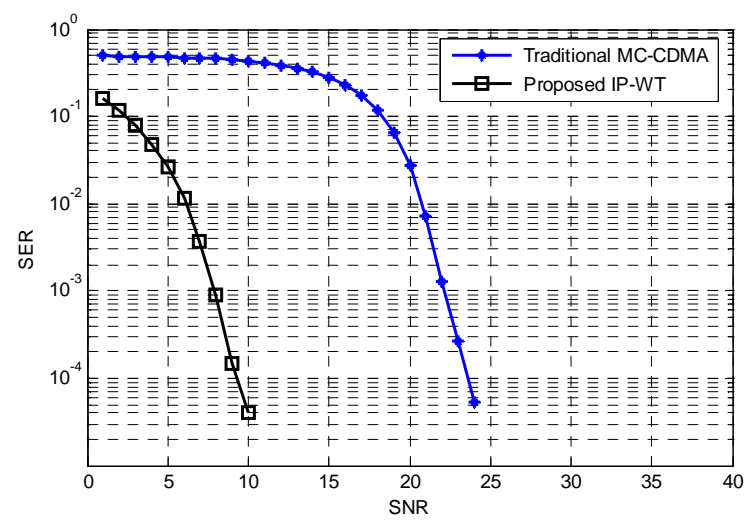

Figure 4. Performance of the proposed and traditional model in AWGN channel

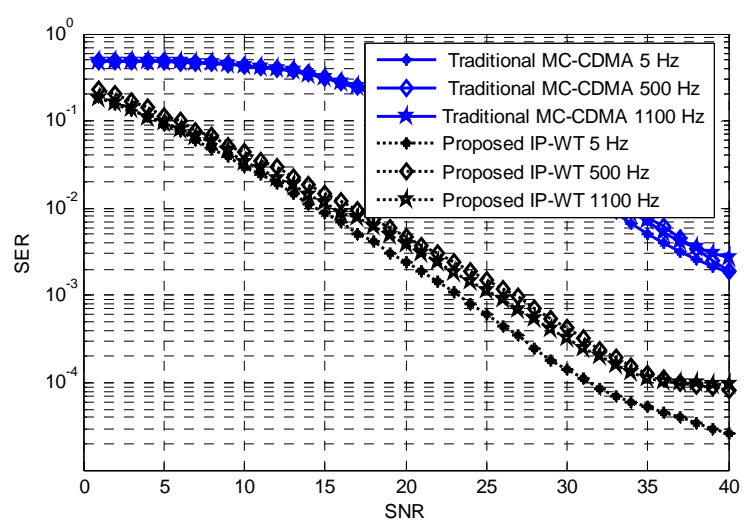

Figure 5. Performance of the proposed and traditional model in flat fading channel (Doppler frequencies are: 5, 500, and $1100 \mathrm{~Hz})$

\subsection{Performance of the Proposed System in Selective Fading Channel}

In this type of channel, the frequency components of the transmitted signal are affected by uncorrelated changes, where the parameters of the channel in this case correspond to multipath, the two paths chosen are, the Line Of Sight (LOS) and second path (reflected path). In selective fading channel, many models have been taken to compare the BER performance of the systems, the influence of the attenuation, delay and maximum Doppler shift of the echo is successfully discussed. First, the Doppler shift 
parameter has been taken out of interest; set the Doppler shift to 5 $\mathrm{Hz}, 500$, and $1100 \mathrm{~Hz}$. The path delay has been set to 1 sample and the path gain to $-8 \mathrm{~dB}$.

From Fig. (6), it can be seen at Doppler frequency $=5 \mathrm{~Hz}$, the proposed MC-CDMA has $\mathrm{SNR}=21 \mathrm{~dB}$ at $\mathrm{BER}=10^{-3}$ compared with $34 \mathrm{~dB}$ for the original MC-CDMA, this means a gain of about $13 \mathrm{~dB}$ was obtained by the new way over the original system. As the Doppler frequency increases, the BER will increase for both systems and the same value of gain can be obtained by the proposed model as shown in the same figure. Small loss is appeared as the SNR increased to more than $36 \mathrm{~dB}$ for the proposed model.

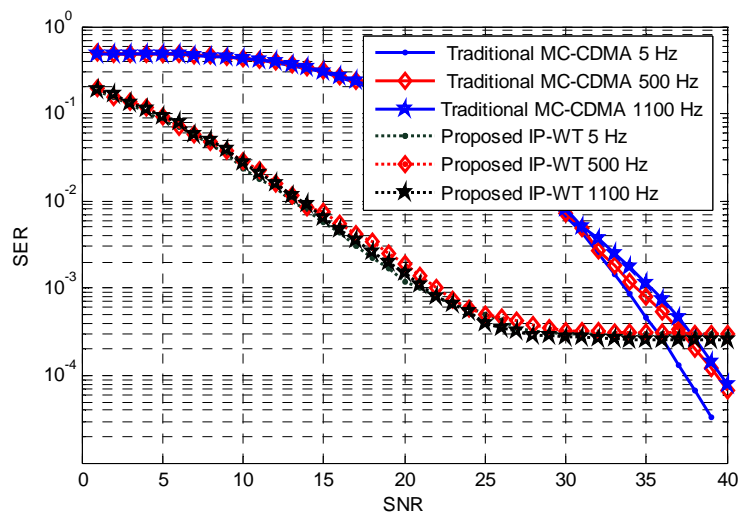

Figure 6. Performance of the proposed and traditional model in selective fading channel (Doppler frequencies are: 5, 500, and $1100 \mathrm{~Hz}$, path delay=1 sample, path gain=-8 dB)

The efficiency of MC-CDMA transceiver is widely affected by increasing or decreasing the cyclic prefix length. If the cyclic extended symbol is used, then, it can provide multipath immunity as well as symbol synchronization tolerance. The transmitted energy increases with the length of guard interval $T_{g}$ of the cyclic prefix, while the received samples and signal remain the same. In this section, the effect of increasing the user specific code length on the performance of the proposed system is studied under the selective fading channel, Doppler frequency $=5 \mathrm{~Hz}$, the number of subcarriers or the FFT size $=32$ bit and 128 bit, the path delay and the path gain for the reflected path are equal to 1 sample and -10 $\mathrm{dB}$, without zero padding and 8 bit cyclic prefix extension is used for FFT models in this simulation.

The performance of the proposed and the traditional models is shown in fig. (7). From this figure, the performance of the proposed model at BER $=10^{-4}$ is high improved as the length of Walsh code increased from 32 bit to 128 bit, while the losses increased by $5 \mathrm{~dB}$ for the traditional MC-CDMA, this property makes the suggested model to be suitable for working in multiple users, since every user has a user specific code, so for large number of users such as 1024 then the size of FFT must be increased to cover the range of the number of users.

\section{CONCLUSIONS}

The simulation of the proposed and traditional MC-CDMA systems has been investigated. It has been shown that the new algorithm is widely active to work under different channel characteristics. Approximately a gain of $13.5 \mathrm{~dB}$ or more was obtained at the AWGN channel, $18 \mathrm{~dB}$ gain was obtained for flat fading channel, and $13 \mathrm{~dB}$ at frequency selective fading channel for different Doppler frequencies. A gain of $18 \mathrm{~dB}$ appeared at selective fading channel for a $\mathrm{BER}=10^{-4}$ from the proposed MCCDMA as the user length code increased from 32 bit to 128 bit.

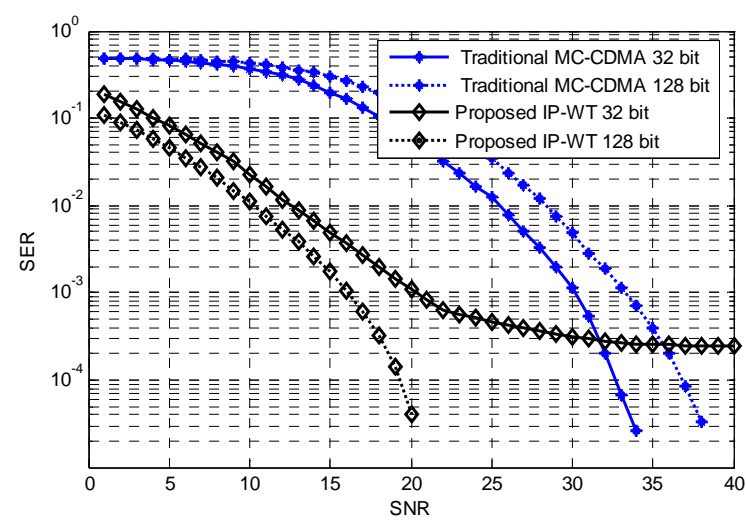

Figure 7. Performance of the proposed and traditional model in selective fading channel (Doppler frequencies are: $5 \mathrm{~Hz}$, path delay=1 sample, path gain=-10 dB, code length=32, and 128 bit)

\section{REFERENCES}

[1] Laith Ali Abdul-Rahaim. "Design and Simulation of STBC(OFDM and CDMA) Transceivers Based on Hybrid Transforms," Ph.D. Thesis, University of Technology/Iraq, January (2007).

[2] Hongbing Zhang, "Wavelet Packet Based Multicarrier CDMA wireless Communication Systems," Ph.D. Thesis, University of Cinicinnati, March, (2004).

[3] Negash B. G. and Nikookar H. "Wavelet Based OFDM for Wireless Channels," International Research Center for Telecommunications Transmission and Radar, Faculty of Information Technology and Systems, Delft University of Technology, (2001).

[4] Nievergelt Yves, "Wavelets Made Easy,” Birkhauser, Boston Inc, April (1999).

[5] Saad Nihad Abdul-Majeed, "A Multiwavelet Based MCCDMA System,” Ph.D. thesis, College of Engineering, BaghdadUniversity, 2006.

[6] MUSTAFA S. A., "Design and Implementation of Orthogonal Frequency Division Multiplexing Transceiver on TMS320C6711,” Ph.D. thesis, College of Engineering, Baghdad University, 2004.

[7] Salih M. Salih, N. Uzunoglou, A. A. Ali, and Waleed A. Mahmoud, "Performance Improvement of MC-CDMA Based FFT," Journal of Applied Electromagnetism of the Trans Black Sea Union of Electromagnetism, in press. 